\title{
The dynamics of humoral immune responses following SARS-CoV-2 infection and the potential for reinfection.
}

\author{
Paul Kellam ${ }^{1,2}$ and Wendy Barclay ${ }^{1}$ \\ ${ }^{1}$ Department of Medicine, Division of Infectious Diseases, Imperial College London, London, \\ SW7 2AZ, UK. \\ ${ }^{2}$ Kymab Ltd., The Bennet Building (B930), Babraham Research Campus, Cambridge, CB22 \\ $3 A T, U K$
}

\begin{abstract}
SARS-CoV-2 is a novel coronavirus that is the causative agent of Coronavirus infectious disease 2019 (COVD-19). As of the $17^{\text {th }}$ April 2020, it has infected 2114269 people resulting in 145144 deaths. The timing, magnitude and longevity of humoral immunity is not yet understood for SARS-CoV-2. Nevertheless, understanding this is urgently required to inform the likely future dynamics of the pandemic, to guide strategies to allow relaxation of social distancing measures and to understand how to deploy limiting vaccine doses when they become available to achieve maximum impact. SARS-CoV-2 is the seventh human coronavirus to be described. Four human coronaviruses circulate seasonally and cause common colds. Two other coronaviruses, SARS and MERS, have crossed from animal sources into humans but have not become endemic. Here we review what is known about the human humoral immune response to epidemic SARS CoV and MERS CoV and to the seasonal, endemic coronaviruses. Then we summarize recent, mostly non-peer reviewed studies into SARSCoV-2 serology and reinfection in humans and non-human primates and summarize current pressing research needs.
\end{abstract}

Keywords: COVID-19; SARS-CoV-2; immune response; antibodies

\section{Serological decline after MERS CoV and SARS CoV infection}

A few studies have assessed antibody titres to MERS CoV and SARS CoV in the months and years following primary infection. Robust immune responses with long lived ( $>1$ year) functional antibodies were seen following severe MERS CoV infections or in those people with prolonged virus shedding (Choe et al., 2017); (Alshukairi et al., 2016). This was also observed in a small study of MERS CoV infections, where neutralizing antibodies were detectable in 6 (86\%) of 7 persons who had previously had severe MERS (including 5 with pneumonia) for at least 34 months after infection. However, in this small group there was evidence of antibody waning; $4 / 7$ showed 4 to 16 -fold reduction in nucleocapsid binding titres and $4 / 7$ show a 2 fold reduction in neutralising titres over 34 months, with $4 / 7$ assessed as having a low neutralising tires throughout (Payne et al., 2016). After mild or asymptomatic MERS CoV infections, antibody responses were either limited or rapidly declined. Although numbers are small, no neutralizing antibody response was seen in 4/6 (Choe et al., 2017)) and $3 / 6$ ((Okba et al., 2019) mild MERS CoV infections for some, not even immediately after infection (Choe et al., 2017). In a separate study of 280 contacts of 26 confirmed MERS CoV index cases, 12 contacts likely to have been infected were identified. $7 / 12$ contacts sampled within 4-14 days of index contact were virus genome positive by RT-PCR but serologically negative (actively infected), whereas $5 / 7$ were virus genome negative, but had detectable binding and neutralising antibody titres (infected and recovered) (Drosten et al., 2014).

Similarly, although SARS CoV was largely associated with symptomatic disease, antibodies decline over time. In a 3 year follow-up of hospitalised SARS CoV patients, SARS CoV IgG binding titres were undetectable in $19.4 \%$ of people by 30 months post infection and neutralizing tires were undetectable in $11.1 \%$ of people at this time (Cao et al., 2007). Consistent with this observation, a study of 98 SARS patients over 2 years showed all had detectable antibody binding titres over 2 
years but that, in a subset, titres declined over this period. 18 individuals with neutralizing antibodies had titres that peaked on day 30 , then decayed gradually so that by 2 years $1 / 18$ had no detectable neutralizing antibodies, and the remaining patients had low antibody titres close to background levels (Mo et al., 2006). Similarly, in a study following 176 previously SARS CoV infected people, the ELISA optical densities (ODs) that indicate antibody titre reduced by $33 \%$ within one year, $46 \%$ by 2 years and $~ 75 \%$ by 3 years (Wu et al., 2007). Nevertheless, other long term follow up studies of SARS CoV show that although antibody titres decline over over 2 (Liu et al., 2006) and 3 years (Liu et al., 2011), neutralizing activity was present in $89 \%(17 / 19)$ of the recovered patients at 36 months, although the the ability of sera to neutralize virus declined from $96 \%$ inhibition at month 3 to $48 \%$ at month 36 (Liu et al., 2011). Although antibody tires to SARS CoV can be detected in people 12 years after infection, over $70 \%$ the people studied $(n=20)$ have extremely low titres (Guo et al., 2020), therefore at 3 and 12 years post infection SARS CoV antibody titres are likely to be very limited for virus neutralisation with little or no ability to protect a person from reinfection, however this requires experimental determination.

Although limited in size, studies of MERS and SARS CoV indicate total binding antibodies and neutralising antibodies decrease to a level where by 2-3 years everyone previously infected will have minimal detectable antibody response, but with those suffering more severe disease have the highest titre antibody responses for longer. Although the time dependent decay of neutralizing antibody titers implies a lack of protection from reinfection to MERS and SARS CoV, this cannot be concluded unequivocally, due to lack of epidemic spread allowing reinfection. It is, however, suggestive of the potential for a population level reduction in protection from reinfection by epidemic CoVs over a short period of time, dependent in some on initial disease severity.

\section{Seroconversion rates to seasonal human coronaviruses}

One indication of the strength of immune protection from coronavirus infection is to consider what is known for the endemic seasonal CoVs, namely the genetically related alphacoronaviruses NL63 and 229E, and the genetically related betacoronaviruses HKU1 and OC43. There is some evidence for antigenic cross protection between the Human CoVs in the same genetic group. A cross sectional seroprevalence study for seasonal human alphacoronaviruses NL63 or $229 \mathrm{E}$, showed $75 \%$ and $65 \%$ of children in the age group 2.5-3.5 years are seropositive for NL63 and 229E respectively, and most children are seropositive by 6 years (Dijkman et al., 2008). In adults, respiratory infection by human seasonal CoVs accounted for 22\% (43/195) (Gorse et al., 2020) and 25\% (Ambrosioni et al., 2014) acute respiratory illness. Therefore, the ability of human seasonal coronaviruses to infect adults who have likely been infected as children can be accounted for either by, virus escape from neutralization (drift), reinfection with a heterologous CoV of a different genotype (alpha- followed by betacoronavirus infections, or vice versa) due to lack of cross protective antibodies, or reinfection with homologous coronavirus due to a sub-protective or waning antibody responses.

The lack of extensive time resolved virus genetic data and a lack of extensive serology studies against extant and historic strains of the 4 seasonal coronaviruses makes the contribution of virus genetic drift to escape from pre-existing protective immune response difficult to judge. However, genetic drift mapping to sugar binding domains in S protein of CoV OC43 suggesting drift may contribute to persistence of this genotype in the human population (Ren et al., 2015). Similar studies on other endemic CoV genotypes are lacking. Therefore, in the absence of drift, and bearing in mind there are only identify 4 genotypes of CoV endemic in humans which account for an esitmated $20 \%$ clinical colds and likely many more asymptomatic infections each year, we can infer that each person becomes infected at least once every 5 years by a coronavirus, and so homologous reinfection must take place over the lifetime of all people.

\section{Reinfection by seasonal human coronaviruses in the community}


A small number of studies have attempted to detect reinfection by endemic CoVs in the community. In a cohort study of community acquired and childhood pneumonia admissions to hospital in Kenya, reinfections by human coronavirus NL63 were detected over a 6 month period (Dec-May 2010) in 46 of 163 patients (28\%) (Kiyuka et al., 2018). Most reinfections resulted in low virus titres and decreased disease. However, for a small number (11\%), reinfection resulted in higher virus shedding compared to the previous infection, with the caveat that the peak viral genome load in the first infection could have been missed in the sampling window. When reinfections occurred up to 80 days after first infection, the secondary infection virus load was usually low. However, reinfection after 80 days could result in high viral genome load, compatible with such viruses being capable of onwards transmission. Sequence analysis of paired viral samples from the same individual reinfected after 80 days suggested reinfection was by a homologous CoV (Kiyuka et al., 2018), however, no antibody levels were measured in this study.

In a recent population study from the FLUWATCH project, over 5 seasons 2006-7 to 2010-11, the seasonal CoVs NL63, 229E and OC43, were detected at a rate of 390 infections ( $95 \% \mathrm{Cl} 338-448$ ) per 100,000 person-weeks. The rates of infection stratified by age showed a bimodal distribution with peaks at ages $0-4$ and ages $16-44$ consistent with previous serology studies. Importantly, 8 subjects had more than one consecutive coronavirus infection. Of these, no participants had the same coronaviruses strain twice, with modelling suggesting this provides some evidence for lasting immunity. Nonetheless, analysis of the CoV infection pairs per person shows these small numbers partition into $4 / 8$ having a reinfection within $7-15$ weeks whereas $4 / 8$ have a reinfection between 23-56 weeks. The former group all comprise infection-reinfection with heterologous alpha- (NL63 or 229E) and beta- (OC43) CoVs pairs, consistent with lack of serological cross protection, whereas $3 / 8$ of the latter group had homologous reinfection of alphacoronaviruses (Aldridge et al., 2020). Although too small in numbers to be definitive, this suggests that serological protection from reinfection does exist but that it declines over a year, when infection with a virus of the same genotype becomes possible.

Evidence to support seroprotection against homologous virus genotypes exists in children, using serology assays specific for the carboxyl-terminal region of the nucleocapsid protein of each of the four viruses. Seroconversion to NL63 (alphacoronavirus) and OC43 (betacoronavirus) occurs more frequently in children in both households and in hospitals. When examining small numbers of reinfections, seroconversion to NL63 was correlated with protection from infection by 229E, both alphacoronaviruses. Similarly, seroconversion to OC43 can protect from reinfection by HKU1, both betacoronaviruses. However, the reciprocal protection (229E protects against NL63 and HKU1 against OC43) did not occur (Dijkman et al., 2012), suggesting that even homologous protection by genetically related CoV is not immunologically simple. Recently, transmission dynamic modelling of OC43 and HKU1 in the USA based on weekly laboratory testing for both viruses showed peak winter infections occurring each year for OC43 but every two years for HKU1. Using susceptible, exposed, infected, recovered, susceptible (SEIRS) best fit models suggested immunity to both viruses remains for 45 weeks and that immunity to OC43 provides stronger cross immunity to HKU1 than the reverse, consistent with Dijkman et al (Kissler et al., 2020).

\section{Reinfection by seasonal human coronaviruses in controlled human infection models (CHIM).}

Another way to distinguish between infection due to virus escape from neutralization, including heterologous challenge or infection in the presence of sub-protective antibody responses, is to attempt to experimentally infect adult volunteers with seasonal human coronavirus either in the presence of their preexisting immunity or by re-challenge with a homologous virus. Inoculation of healthy adult volunteers with the endemic coronavirus $229 \mathrm{E}$ led to infection in 10/15 people and clinical symptoms in 8 of those 10 infected people, even though most must have already experienced $229 \mathrm{E}$ infection previously in their lives. All those infected had increased antibody titres 
within 3 weeks of infection, which rapidly declined by 12 weeks and returned to baseline by 52 weeks. When re-challenged at 1 year, 66\% (6/9) became reinfected, but none developed clinical symptoms (Callow et al., 1990). There is no data about the levels of virus shedding after the first or second challenge. These data were different to earlier studies where reinfection by a homologous coronavirus after 1 year did not occur, but reinfection with heterologous virus produced symptoms of infection. However, in the absence of sequence information about these heterologous '229E-like' CoVs and the possibility that Reed's volunteers were more robustly infected initially, with higher antibody titres taking longer to decay, these data are not easy to interpret (Reed, 1984).

\section{Different tests to measure SARS CoV2 antibodies}

Antibody responses to SARS-CoV-2 infection in humans and animal models have been reported in very recently published papers and non-peer reviewed preprints. These early studies suggest the immune response to SARS-CoV-2 is similar to that for SARS-CoV and MERS-CoV. Most infected individuals (RT-PCR positive) seroconvert 10-14 days after symptom onset, but antibody levels in some mild cases can be low or undetectable. There is no data at all as yet on how long the antibodies remain and what level of antibody is associated with immune protection. In comparing studies, caution should be exercised however, because many of the studies use different assays to measure the serological response and these are not yet calibrated against each other nor shown to have sufficient sensitivity and specificity to address all serological questions.

The gold standard test for antiviral antibodies is the virus neutralization test. This measures if antibodies in a serum sample can prevent susceptible cells from being infected when the antibody is mixed with a standard challenge dose of virus. However, using this test for SARS-CoV-2 requires work inside high containment laboratories using infectious virus. A surrogate neutralization assay uses pseudotyped virus particles (PV) that bear the Spike protein of the SARS-CoV-2 virus. PV neutralisation assays can be performed at lower containment levels and are read out with a suitable reporter such as luciferase, meaning they should be scalable. Immunofluorescent tests (IF) also use virus-infected cells but detect the presence of antibodies in the sample through their reaction with viral antigens expressed in the fixed cells without assessment of the functionality of the antibodies.

Alternatively, enzyme linked immunosorbent assays (ELISA) tests and lateral flow assays, which also do not measure the function of the antibody, detect binding to a given antigen. The antigen is usually a recombinant protein such as whole Spike protein, although some tests use just the $\mathrm{N}$ terminal spike subdomain (S1), the $\mathrm{N}$ terminal domain of Spike, and others only use the receptor binding domain (RBD). It is possible that the smaller the spike fragment used, the less likely it is that antibodies in the sera raised against other endemic human coronaviruses will cross react. However, a recent study comparing 3 CE-marked commercial ELISA assays and 6 point of contact (POC) tests that were available in Denmark showed the limitations of current serologic assays (Lassaunière et al., 2020). Thirty serum samples from severe COVID patients were assessed, along with 10 negative sera and another 71 sera from people with other viral infections to test for specificity. The Wantai SARS CoV2 total antibody ELISA that has Spike RBD as the antigen was the most sensitive test, $100 \%$ of day 10 samples were positive. The Euroimmun IgG test was less sensitive and only detected $78 \%$ of the same samples. In addition, the Euroimmun IgG ELISA showed poor specificity because it detected antibodies in 3 sera from patients not infected by SARS-CoV-2. Rapid improvements in SARS-CoV-2 serology are still needed. Nevertheless, individual laboratories have started to use what assays are currently available to understand the immune response to this new human virus.

Antibody responses reported in SARS CoV2 patients.A study of 173 people admitted into hospital in China with acute respiratory infection syndromes and/or abnormalities in chest CT images (Zhao et al., 2020) used three different assays to measure seroconversion. Similar to the Wantai commercial test above, one measured total antibody to the Spike receptor binding domain (RBD), the second 
measured IgM to the same Spike RBD antigen and the third assay measured IgG against nucleoprotein $(\mathrm{N})$. The first assay detected positive sera in $93.1 \%(161 / 173)$ of patients, with a median response time of 11 days, the second measured a seroconversion rate of $82.7 \%(143 / 173)$, median response time 12 days, but the response rate for IgG to the nucleoprotein was lower at $64.7 \%(112 / 173)$ and took longer to appear, with median response time of 14 days. In later samples collected between 15-39 days from disease onset, the assay that measured Spike RBD antibodies detected seronconversion in 100\% patients, whereas the other assays were less sensitive: RBD IgM in $94.3 \%$ and $\mathrm{N}$ IgG in $79.8 \%$ of patients. Thus, SARS-CoV-2 seroconversion occurs on a time course that is consistent with other epidemic CoVs and antibodies to Spike RBD were the most reliable for case counting in this study. At 2 weeks post symptom onset, antibody titres were statistically higher in critical compared to non-critical patients, possibly due to different rates to a maximal antibody response or reflecting similar disease severity observations from MERS-CoV and SARS-CoV patients as described above (Zhao et al., 2020). A large cohort serology study of 285 COVID-19 positive patients of which 262 had a record of disease symptoms, and 39 were severe infections from 3 hospitals in Hubei province determined the antibody response to nucleoprotein and a peptide from spike protein of SARS-CoV-2. This showed that all patients seroconverted by 17-19 days after symptom onset and that severely ill patients had a significantly higher IgG titre compared to nonsevere cases after 7-14 days post symptom onset, but that by 15-21 days there was no difference in the mean antibody tire between these groups. However, a considerable range of antibody tires from low to high was clearly seen in the non-severe group and that IgG titers entered a plateau within 6 days after the first positive samples (Long et al., 2020). Similar results continue to accumulate in other serological studies from China (Lou et al., 2020).

In a separate European collaborative study, in-house and commercial ELISAs together with a virus neutralization assay were used to measure antibodies in a total of 19 severe and mild cases. A temporal study of seroconversion in three patients showed the patient with severe disease became antibody positive earlier than the other two patients who had mild disease, indeed, one mild patient only gave a positive serum sample using the nucleocapsid ELISA or the neutralization test and only at 28 days after symptom onset (Okba et al., 2020). Further, in 9 mild cases from early in the German outbreak, antibody responses were measured by neutralization assay and by immunofluorescence detecting IgG and IgM binding antibodies. There was incomplete correlation between the titres using different assays, with seroconversion occurring by day 7 in $50 \%$ patients and in all patients by 14 days after symptom onset. The onset of the antibody response however, did not result in a rapid decline in virus shedding (Wölfel et al., 2020). In contrast, the timing and functionality of the immune response to SARS-CoV-2 infection was considered in a detailed study of a single female patient with moderate disease in Australia. The appearance of antibody secreting cells, $T$ follicular helper cells and CD8 positive T cells in the blood of this patient at day 7-9 was coincident with a fall in virus titre and recovery (Thevarajan et al., 2020). The antibody response was also investigated in 23 patients in Hong Kong (To et al., 2020; Wu et al., 2020), showing excellent the correlation between virus neutralisation activity and IgG titres to nucleoprotein and the S1 RBD were excellent. Antibody trajectories over 20 days from this small number of severe and mild cases again demonstrate variability in individual early antibody responses, that in this study did not correlate with disease severity. A further large study of a recovered cohort of 175 patients in Shanghai, measured neutralizing antibody titres by the ability of sera to block pseudotyped virus (PV) entry, along with other serology assays (Wu et al., 2020). The average time for seroconversion was 10-15 days. The typical pattern observed was that patients with more severe illness showed higher neutralising antibody (NAb) titres. Importantly, in this study around $30 \%$ patients showed very low Nab titres, and 10 patients (6\%) who were confirmed to have been infected from having an RT-PCR positive respiratory sample did not show any antibody response at all even at a later time point 2 weeks after hospital discharge. In the positive samples taken 2 weeks after hospital discharge there 
was no evidence of antibody waning. Given the variation in NAb titres in it will be important to screen convalescent plasma if it is to be used for prevention or treatment.

Considerably more, well controlled, serological studies are needed, with more focus on how antibody binding assays correlate with measures of serological protection from virus infection and reinfection, such as virus neutralisation assays. To test for specificity of antibody assays, sera collected from individuals in 2019 or earlier can be screened, and to test for sensitivity, sera from people in whom SARS-CoV-2 infection has been confirmed by RT-PCR can be assayed. In one such study, neutralising antibodies measured by the PV assay were not detected in sera taken in 2019 from 100 blood donors from Scotland, nor in 500 samples from Scotland in mid-March, but were detected in 5 of 500 Scottish sera taken in late March. An ELISA assay using S antigen detected all 5 positive sera and also one additional sample from the later sample set (Thompson et al., 2020). The PV neutralization assay has been used to measure potent antibodies raised in rats immunized with a potential SARS CoV2 vaccine based on the spike protein RBD fragment. The antibodies were as potent at inhibiting PV entry as ACE2- Ig, a decoy receptor molecule and potent SARS CoV2 entry inhibitor (Quinlan et al., 2020). This suggests that PV neutralization will be a good correlate for protective antibodies in vaccine studies, but further studies using whole virus neutralization will be needed to confirm this.

\section{Studies on SARS-CoV2 antibodies in experimental animal infections.}

Animal studies can provide a bridge to understanding serology and protection from infection and several species are now known to be susceptible to SARS CoV-2 infection including non-human primates, ferrets and cats (Chen, 2020; Kim et al., 2020; Munster et al., 2020). Infected ferrets had serum neutralizing antibodies at 12 days post infection, but so far no re-challenge experiments were reported (Chen, 2020). Rhesus macaques are susceptible to SARS-CoV-2, where infection causes a respiratory disease lasting 8-16 days, with detectable high viral loads in the nose, throat and bronchoalveolar lavages. All animals seroconverted to the Spike protein and showed neutralising antibodies by 10 days post infection (Munster et al., 2020). In another study in rhesus macaques, two animals were rechallenged with virus at 28 days from the primary infection, when Anti-Spike antibodies were detectable and neither became infected (Bao et al., 2020). This is unsurprising as the animals were most likely at or near the peak of their seroconversion but suggests that immediate reinfection in the face of robust neutralising antibodies to SARS-CoV-2 is not possible.

\section{Conclusion}

It is clear that most people infected with SARS-CoV-2 display an antibody response between 10 and 14 days after infection. In some mild cases, detection of antibodies requires a long time after symptoms, and in a small number of cases, antibodies are not detected at all, at least during the time scale of the reported studies. There is a paucity of information about the longevity of the antibody response to SARS-CoV-2, but it is known that antibodies to other human coronaviruses wane over time, and there are some reports of reinfection with homologous coronaviruses after as little as 80 days. Thus, reinfection of previously mild SARS-CoV-2 cases is a realistic possibility that should be considered in models of a second wave and the post pandemic era (Kissler et al., 2020). Obtaining longitudinal serological data where both binding titres and functional neutralisation titres stratified by age groups and previous disease severity status should be undertaken as a matter of urgency. Further, people with low antibody titres after mild disease should be followed up for evidence of reinfection and recurrent disease by regular clinical monitoring and diagnostic virus detection by RT-PCR. If reinfection is detected, serial viral load and measures of antibody status at the time of reinfection should be established. Detailed human immunology characterisation and animal studies will be necessary to determine if prior infection leads to an altered disease course if reinfection occurs. It is possible and likely that protective mechanisms through other arms of the immune response (memory and cytotoxic T cells) and alter the COVID-19 disease course upon 
reinfection either by diminishing symptoms in the absence of protective antibodies, or enhancing infection at the nadir of the humoral immune response by sub-neutralising antibody titres. It is also unclear if reinfections will result in onward transmission, but that cannot be excluded. Recent modelling studies, however, suggest that waning humoral immunity could have a major impact in the course of SARS-CoV-2 becoming 5th endemic human coronavirus. Under the assumptions of waning immunity across the population of the USA, similar to OC43 and HKU1, models show that if immunity is not permanent many epidemiological scenarios lead to SARS-CoV-2 becoming a seasonal human coronavirus, with either annual, biennial or sporadic patterns of epidemics over the next five years (Kissler et al., 2020). This means susceptible, exposed, infected, recovered, susceptible (SEIRS) models will need to replace susceptible, infected, recovered (SIR) models to inform strategies to exit from the current policies of complete transmission suppression. Serological studies will need to supply data for parameter estimates in these models as well as inform vaccine deployment to achieve maximum effects when any initial supplies of vaccine will be limited. If SARSCoV- 2 immunity can be engineered to be permanent by regular vaccination models suggest that SARS-CoV-2 infection can be dramatically reduced or possibly eliminated (Kissler et al., 2020).

\section{Conflicts of Interest}

The authors have no conflicts of interests with respect to this review. 


\section{Figure legends}

Figure 1. A schematic representation of the SARS-CoV-2 immune response following infection. Seroconversion occurs from approximately 10 days after symptom onset with the exact timing of IgM (blue line) and IgG (red line) appearance presently unclear, but with a suggestion the IgM occurs at the time of and overlapping with the IgG response. The IgG antibody titres rise from day 10 onwards to reach a peak whose height is likely to be influenced, on a case by case basis, by disease severity and virus load. The level of antibody protection from reinfection (black dotted line), the duration of the total humoral immune response above this level, and the rate of decline from mild or severe infection induced antibodies is not known for SARS-CoV-2. Similarly, the proportion of infected individuals that do not mount a protective immune response (green line) is not known. 


\section{References}

Aldridge, R. W., Lewer, D., Beale, S., Johnson, A. M., Zambon, M., Hayward, A. C., Fragaszy, E. \& Flu Watch Group. (2020). Seasonality and immunity to laboratory-confirmed seasonal coronaviruses (HCoV-NL63, HCoV-OC43, and HCoV-229E): results from the Flu Watch cohort study [version 1; peer review: awaiting peer review]. Wellcome Open Res 5, 52.

Alshukairi, A. N., Khalid, I., Ahmed, W. A., Dada, A. M., Bayumi, D. T., Malic, L. S., Althawadi, S., Ignacio, K., Alsalmi, H. S. \& other authors. (2016). Antibody response and disease severity in healthcare worker MERS survivors. Emerging Infect Dis 22.

Ambrosioni, J., Bridevaux, P. O., Wagner, G., Mamin, A. \& Kaiser, L. (2014). Epidemiology of viral respiratory infections in a tertiary care centre in the era of molecular diagnosis, Geneva, Switzerland, 2011-2012. Clin Microbiol Infect 20, 0578-84.

Bao, L., Deng, W., Gao, H., Xiao, C., Liu, J., Xue, J., Lv, Q., Liu, J., Yu, P. \& other authors. (2020). Reinfection could not occur in SARS-CoV-2 infected rhesus macaques. BioRxiv.

Callow, K. A., Parry, H. F., Sergeant, M. \& Tyrrell, D. A. (1990). The time course of the immune response to experimental coronavirus infection of man. Epidemiol Infect 105, 435-446.

Cao, W.-C., Liu, W., Zhang, P.-H., Zhang, F. \& Richardus, J. H. (2007). Disappearance of antibodies to SARS-associated coronavirus after recovery. N Engl J Med 357, 1162-1163.

Chen, H. (2020). Susceptibility of ferrets, cats, dogs, and different domestic animals to SARScoronavirus-2. BioRxiv.

Choe, P. G., Perera, R. A. P. M., Park, W. B., Song, K.-H., Bang, J. H., Kim, E. S., Kim, H. B., Ko, L. W. R., Park, S. W. \& other authors. (2017). MERS-CoV Antibody Responses 1 Year after Symptom Onset, South Korea, 2015. Emerging Infect Dis 23, 1079-1084.

Dijkman, R., Jebbink, M. F., El Idrissi, N. B., Pyrc, K., Müller, M. A., Kuijpers, T. W., Zaaijer, H. L. \& van der Hoek, L. (2008). Human coronavirus NL63 and 229E seroconversion in children. J Clin Microbiol 46, 2368-2373.

Dijkman, R., Jebbink, M. F., Gaunt, E., Rossen, J. W. A., Templeton, K. E., Kuijpers, T. W. \& van der Hoek, L. (2012). The dominance of human coronavirus OC43 and NL63 infections in infants. J Clin Virol 53, 135-139.

Drosten, C., Meyer, B., Müller, M. A., Corman, V. M., Al-Masri, M., Hossain, R., Madani, H., Sieberg, A., Bosch, B. J. \& other authors. (2014). Transmission of MERS-coronavirus in household contacts. N Engl J Med 371, 828-835.

Gorse, G. J., Donovan, M. M. \& Patel, G. B. (2020). Antibodies to coronaviruses are higher in older compared with younger adults and binding antibodies are more sensitive than neutralizing antibodies in identifying coronavirus-associated illnesses. J Med Virol 92, 512-517.

Guo, X., Guo, Z., Duan, C., chen, Z., Wang, G., Lu, Y., Li, M. \& Lu, J. (2020). Long-Term Persistence of IgG Antibodies in SARS-CoV Infected Healthcare Workers. medRxiv.

Kim, Y.-I., Kim, S.-G., Kim, S.-M., Kim, E.-H., Park, S.-J., Yu, K.-M., Chang, J.-H., Kim, E. J., Lee, S. \& other authors. (2020). Infection and Rapid Transmission of SARS-CoV-2 in Ferrets. Cell Host Microbe.

Kissler, S. M., Tedijanto, C., Goldstein, E., Grad, Y. H. \& Lipsitch, M. (2020). Projecting the transmission dynamics of SARS-CoV-2 through the postpandemic period. Science.

Kiyuka, P. K., Agoti, C. N., Munywoki, P. K., Njeru, R., Bett, A., Otieno, J. R., Otieno, G. P., Kamau, E., Clark, T. G. \& other authors. (2018). Human coronavirus NL63 molecular epidemiology and evolutionary patterns in rural coastal kenya. J Infect Dis 217, 1728-1739.

Lassaunière, R., Frische, A., Harboe, Z. B., Nielsen, A. C., Fomsgaard, A., Krogfelt, K. A. \& Jørgensen, C. S. (2020). Evaluation of nine commercial SARS-CoV-2 immunoassays. medRxiv.

Liu, L., Xie, J., Sun, J., Han, Y., Zhang, C., Fan, H., Liu, Z., Qiu, Z., He, Y. \& Li, T. (2011). Longitudinal profiles of immunoglobulin $\mathrm{G}$ antibodies against severe acute respiratory syndrome coronavirus 
components and neutralizing activities in recovered patients. Scand J Infect Dis 43, 515-521.

Liu, W., Fontanet, A., Zhang, P.-H., Zhan, L., Xin, Z.-T., Baril, L., Tang, F., Lv, H. \& Cao, W.-C. (2006).

Two-year prospective study of the humoral immune response of patients with severe acute respiratory syndrome. J Infect Dis 193, 792-795.

Long, Q., Deng, H., Chen, J., Hu, J., Liu, B., Liao, P., Lin, Y., Yu, L., Mo, Z. \& other authors. (2020). Antibody responses to SARS-CoV-2 in COVID-19 patients: the perspective application of serological tests in clinical practice. medRxiv.

Lou, B., Li, T., Zheng, S., Su, Y., Li, Z., Liu, W., Yu, F., Ge, S., Zou, Q. \& other authors. (2020). Serology characteristics of SARS-CoV-2 infection since the exposure and post symptoms onset. medRxiv.

Mo, H., Zeng, G., Ren, X., Li, H., Ke, C., Tan, Y., Cai, C., Lai, K., Chen, R. \& other authors. (2006). Longitudinal profile of antibodies against SARS-coronavirus in SARS patients and their clinical significance. Respirology 11, 49-53.

Munster, V., Feldmann, F., Williamson, B., van Doremalen, N., Perez-Perez, L., Schultz, J., MeadeWhite, K., Okumura, A., Callison, J. \& other authors. (2020). Respiratory disease and virus shedding in rhesus macaques inoculated with SARS-CoV-2. BioRxiv.

Okba, N. M. A., Muller, M. A., Li, W., Wang, C., GeurtsvanKessel, C. H., Corman, V. M., Lamers, M. M., Sikkema, R. S., de Bruin, E. \& other authors. (2020). SARS-CoV-2 specific antibody responses in COVID-19 patients. medRxiv.

Okba, N. M. A., Raj, V. S., Widjaja, I., GeurtsvanKessel, C. H., de Bruin, E., Chandler, F. D., Park, W. B., Kim, N.-J., Farag, E. A. B. A. \& other authors. (2019). Sensitive and Specific Detection of Low-Level Antibody Responses in Mild Middle East Respiratory Syndrome Coronavirus Infections. Emerging Infect Dis 25, 1868-1877.

Payne, D. C., Iblan, I., Rha, B., Alqasrawi, S., Haddadin, A., Al Nsour, M., Alsanouri, T., Ali, S. S., Harcourt, J. \& other authors. (2016). Persistence of Antibodies against Middle East Respiratory Syndrome Coronavirus. Emerging Infect Dis 22, 1824-1826.

Quinlan, B. D., Mou, H., Zhang, L., Gao, Y., He, W., Ojha, A., Parcells, M. S., Luo, G., Li, W. \& other authors. (2020). The SARS-CoV-2 receptor-binding domain elicits a potent neutralizing response without antibody-dependent enhancement. BioRxiv.

Reed, S. E. (1984). The Behaviour of Recent Isolates of Human Respiratory Coronavirus In Vitro and in Volunteers. J Med Virol 13, 179-192.

Ren, L., Zhang, Y., Li, J., Xiao, Y., Zhang, J., Wang, Y., Chen, L., Paranhos-Baccalà, G. \& Wang, J. (2015). Genetic drift of human coronavirus OC43 spike gene during adaptive evolution. Sci Rep 5, 11451.

Thevarajan, I., Nguyen, T. H. O., Koutsakos, M., Druce, J., Caly, L., van de Sandt, C. E., Jia, X., Nicholson, S., Catton, M. \& other authors. (2020). Breadth of concomitant immune responses prior to patient recovery: a case report of non-severe COVID-19. Nat Med 26, 453-455.

Thompson, C., Grayson, N., Paton, R., Lourenço, J., Penman, B., Lee, L. N., Odon, V., Mongkolsapaya, J., Chinnakannan, S. \& other authors. (2020). Neutralising antibodies to SARS coronavirus 2 in Scottish blood donors - a pilot study of the value of serology to determine population exposure. medRxiv.

To, K. K.-W., Tsang, O. T.-Y., Leung, W.-S., Tam, A. R., Wu, T.-C., Lung, D. C., Yip, C. C.-Y., Cai, J.-P., Chan, J. M.-C. \& other authors. (2020). Temporal profiles of viral load in posterior oropharyngeal saliva samples and serum antibody responses during infection by SARS-CoV-2: an observational cohort study. Lancet Infect Dis.

Wölfel, R., Corman, V. M., Guggemos, W., Seilmaier, M., Zange, S., Müller, M. A., Niemeyer, D., Jones, T. C., Vollmar, P. \& other authors. (2020). Virological assessment of hospitalized patients with COVID-2019. Nature.

Wu, F., Wang, A., Liu, M., Wang, Q., Chen, J., Xia, S., Ling, Y., Zhang, Y., Xun, J. \& other authors. (2020). Neutralizing antibody responses to SARS-CoV-2 in a COVID-19 recovered patient cohort and their implications. medRxiv. 
Wu, L.-P., Wang, N.-C., Chang, Y.-H., Tian, X.-Y., Na, D.-Y., Zhang, L.-Y., Zheng, L., Lan, T., Wang, L.F. \& Liang, G.-D. (2007). Duration of antibody responses after severe acute respiratory syndrome. Emerging Infect Dis 13, 1562-1564.

Zhao, J., Yuan, Q., Wang, H., Liu, W., Liao, X., Su, Y., Wang, X., Yuan, J., Li, T. \& other authors. (2020). Antibody responses to SARS-CoV-2 in patients of novel coronavirus disease 2019. medRxiv. 


\section{Figure 1}

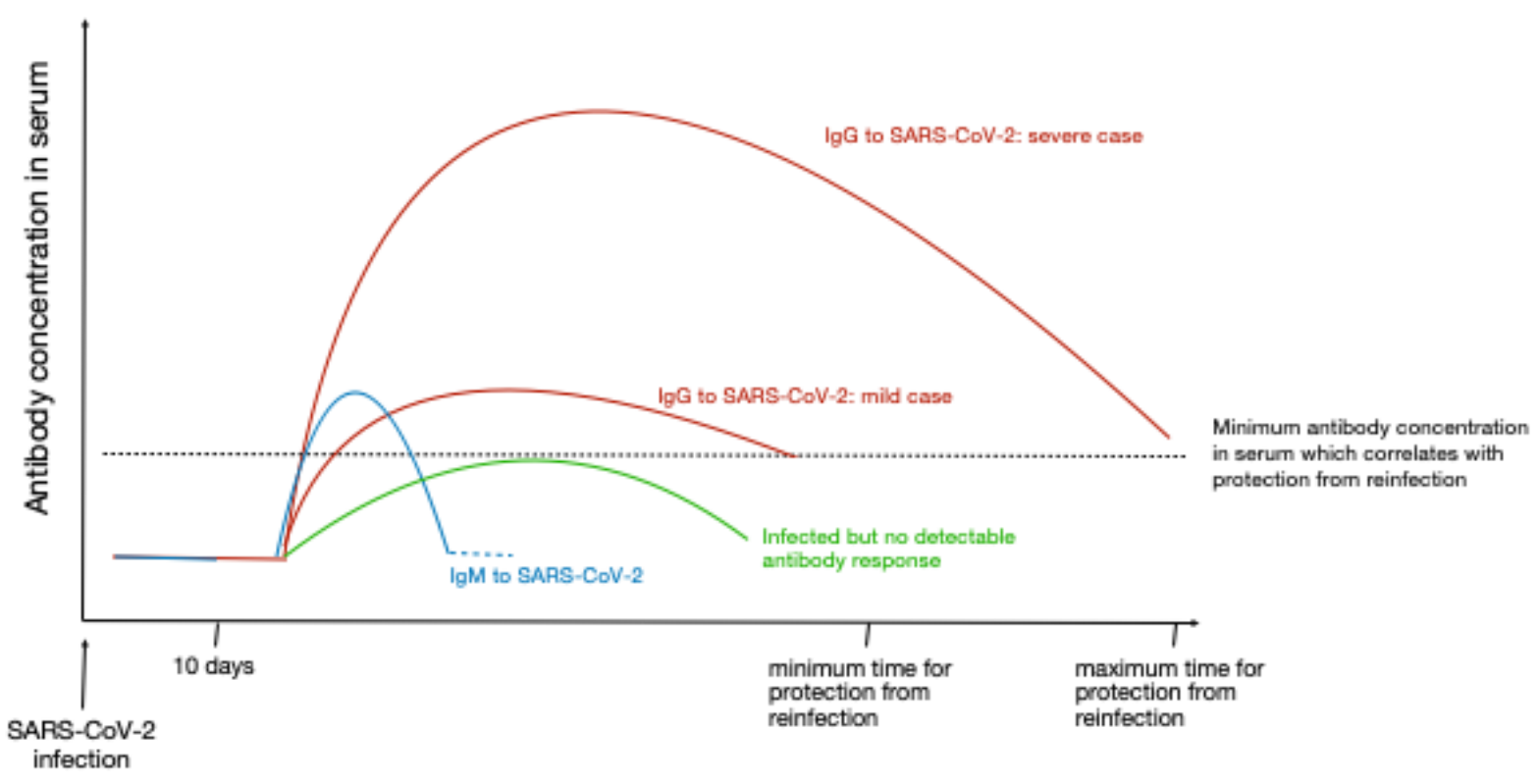

